\title{
NOTE ON THE THERMAL STRESSES IN A LONG CIRCULAR CYLINDER OF $m+1$ CONCENTRIC MATERIALS*
}

\section{By B. E. GATEWOOD (AF Institute of Technology)}

It has been shown that the stresses and displacements in a long cylindrical body ${ }^{1}$ can be expressed in terms of two functions $U$ and $V$ which satisfy the equations

$$
\nabla^{4} U=0, \quad \nabla^{2} V=k T,
$$

where $T$ is the temperature change, and $k=E q \alpha /(q-1)$ with $\mathrm{E}$ Young's modulus, $q$ the reciprocal of Poisson's ratio, and $\alpha$ the linear coefficient of thermal expansion. The stresses are as follows

$$
\begin{gathered}
\sigma_{x}=U_{y y}-V_{y y}, \quad \sigma_{y}=U_{x x}-V_{x x}, \quad \tau_{x y}=V_{x y}-U_{x y} . \\
\tau_{z x}=0, \quad \tau_{y z}=0, \quad \sigma_{z}=E e_{z}-k T+\nabla^{2} U / q,
\end{gathered}
$$

where the constant strain $e_{z}$ is determined from the requirement that the total normal force on the cross-section of the cylinder be zero. The function $U$ can be expressed ${ }^{1}$ in terms of two analytic functions $\phi(z)$ and $H(z)$ as

$$
U=z \bar{\phi} \overline{(z)}+\bar{z} \phi(z)+H(z)+\bar{H} \overline{(z)},
$$

and the function $V$ may be taken as a particular integral of $\nabla^{2} V=k T$ since the solution of $\nabla^{2} V=0$ can be included in $H(z)$.

The boundary conditions for the case of a circular cylinder composed of $m+1$ different materials concentric to each other, each of which has different $E, q$, and $\alpha$, can be obtained by taking the displacements and normal stresses continuous on the junction surfaces and the normal stress zero on the outside surface (let $S_{m}$ be the inside material, $S_{m-1}$ the adjoining material concentric to $S_{m}$, and $S_{0}$ the outside material; let $C_{0}$ of radius $r_{0}$ be the outside boundary and $C_{i}$ of radius $r_{i}$ the boundary between $S_{i}$ and $S_{i-1}$ with $\left.j=1,2, \cdots, m\right)$ :

$$
\begin{aligned}
& \phi_{0}(t)+t \overline{\phi_{0}^{\prime}(t)}+\bar{\psi}_{0} \overline{(t)}=[Y]_{0}, \text { on } C_{0}, \\
& \left(1+M_{i}\right) \phi_{i}(t)+N_{i} \phi_{i-1}(t)+t \bar{\phi}_{i}^{\prime}\left(\overline{t)}+\bar{\psi}_{i} \overline{(t)}\right. \\
& =R_{i} t+[Y]_{i} \text {, on } C_{i} \text { with } j=1,2, \cdots, m, \\
& M_{i} \phi_{i}(t)+\left(1+N_{i}\right) \phi_{i-1}(t)+t \bar{\phi}_{i-1}^{\prime} \overline{(t)}+\bar{\psi}_{i-1} \overline{(t)} \\
& =R_{i} t+[Y]_{i-1} \text {, on } C_{i},
\end{aligned}
$$

where $z=t$ on $C_{i}$, $Y=V_{x}+i V_{\nu}, G=E q / 2(q+1), \psi(z)=H^{\prime}(z)$, and

$$
\begin{aligned}
M_{i} & =4 G_{i-1}\left(q_{i}-1\right) / q_{i}\left(G_{i}-G_{i-1}\right), \quad j=1,2, \cdots, m, \\
-N_{i} & =4 G_{i}\left(q_{i-1}-1\right) / q_{i-1}\left(G_{i}-G_{i-1}\right), \\
R_{i} & =2 e_{2} G_{i} G_{i-1}\left(q_{i-1}-q_{i}\right) / q_{i} q_{i-1}\left(G_{i}-G_{i-1}\right) .
\end{aligned}
$$

${ }^{*}$ Received Sept. 22, 1947.

${ }^{1}$ B. E. Gatewood, Thermal stresses in long cylindrical bodies, Phil. Mag. (7) 32, 282-301 (1941). 
Since the functions $\phi_{i}$ and $\psi_{i}$ are analytic, they can be represented by a Laurent series in $S_{i}(j=0,1, \cdots, m-1)$ and a power series in $S_{m}$ :

$$
\begin{aligned}
& \phi_{i}(z)=\sum_{n=0}^{\infty}\left(a_{i n}+i b_{i n}\right) z^{n}+\sum_{n=1}^{\infty}\left(c_{i n}+i d_{i n}\right) z^{-n}, \\
& \psi_{i}(z)=\sum_{n=0}^{\infty}\left(e_{i n}+i f_{i n}\right) z^{n}+\sum_{n=1}^{\infty}\left(g_{i n}+i h_{i n}\right) z^{-n},
\end{aligned}
$$

with $c_{m n}=d_{m n}=g_{m n}=h_{m n}=0$. Also, $Y_{i}$ may be expressed in a series of powers of $z$ such that on $C_{i}(j=0,1, \cdots, m)$

$$
\begin{aligned}
& {[Y]_{i}=\sum_{n=0}^{\infty}\left(A_{i n}+i B_{i n}\right) t^{n}+\sum_{n=1}^{\infty}\left(C_{i n}+i D_{i n}\right) t^{-n},} \\
& {[Y]_{i-1}=\sum_{n=0}^{\infty}\left(E_{i n}+i F_{i n}\right) t^{n}+\sum_{n=1}^{\infty}\left(G_{i n}+i H_{i n}\right) t^{-n} .}
\end{aligned}
$$

If these expressions be substituted in the boundary conditions (4), (5), and (6) and the resulting expressions together with their conjugates be integrated over the respective circles by use of the Cauchy integral formula, then a system of equations for determining the desired analytic functions is obtained. In some cases the functions can be determined directly while in others they can be obtained by equating coefficients of like powers of $z$. For the general case the coefficients in Eq. (8) can be expressed in terms of the coefficients in Eq. (9) by means of recursion formulas. The coefficients $a_{j 0}+i b_{i 0}$, $e_{i 0}+i f_{i 0}, b_{i 1}$, and $h_{i 1}$ may be neglected since they contribute nothing to the stresses. $a_{i 1}$ and $g_{i 1}$ are determined by

$$
\begin{gathered}
2 a_{01}+g_{01} / r_{0}^{2}=A_{01}, \quad g_{m 1}=0, \\
\left(2+M_{i}\right) a_{i 1}+N_{i} a_{i-1,1}+g_{i 1} / r_{i}^{2}=R_{i}+A_{i 1}, \quad j=1, \cdots, m, \\
M_{i} a_{i 1}+\left(2+N_{i}\right) a_{i-1,1}+g_{i-1,1} / r_{i}^{2}=R_{i}+E_{i 1} .
\end{gathered}
$$

Define $w_{i n}=a_{i n}+i b_{i n}, x_{i n}=c_{i n}+i d_{i n}, y_{i n}=e_{i n}+i f_{i n}, z_{i n}=g_{i n}+i h_{i n}, W_{i n}=$ $A_{i n}+i B_{i n}$, etc., whence $w_{i 2}$ and $z_{i 2}$ are given by

$$
\begin{gathered}
w_{02}+\bar{z}_{02} / r_{0}^{4}=W_{02}, \quad z_{m 2}=0, \\
\left(1+M_{i}\right) w_{i 2}+N_{i} w_{i-1,2}+\bar{z}_{i 2} / r_{i}^{4}=W_{i 2}, \quad j=1, \cdots, m, \\
M_{i} w_{i 2}+\left(1+N_{i}\right) \dot{w}_{i-1,2}+\bar{z}_{i-1,2} / r_{i}^{4}=Y_{i 2} .
\end{gathered}
$$

The remaining coefficients $w_{i n}, x_{i, n-2}, y_{i, n-2}$, and $z_{i n}(n \geq 3)$ are given by $\left(p_{i}=r_{i+1} / r_{i}\right)$

$$
\begin{aligned}
& -M_{i+1} p_{i}^{2 n} w_{i+1, n}+\left[1+M_{i}-\left(1+N_{i+1}\right) p_{i}^{2 n}\right] w_{i n}+N_{i} w_{i-1, n} \\
& -(n-2) r_{i}^{2-2 n} \bar{x}_{i, n-2}\left(1-p_{i}^{2}\right)=W_{i n}-p_{i}^{2 n} Y_{i+1, n}, \\
& -M_{i+1} \bar{x}_{i+1, n-2}+\left[\left(1+M_{i}\right) p_{i}^{2 n-4}-\left(1+N_{i+1}\right)\right] \bar{x}_{i, n-2}+N_{i} p_{i}^{2 n-4} \bar{x}_{i-1, n-2} \\
& \quad+n r_{i+1}^{2 n-2}\left(p_{i}^{-2}-1\right) w_{i n}=p_{i}^{2 n-4} \bar{X}_{i, n-2}-\bar{Z}_{i+1, n-2}, \quad j=1, \cdots, m,
\end{aligned}
$$




$$
\begin{aligned}
& x_{m n}=0, \quad z_{m n}=0, \quad \bar{z}_{0 n}=r_{0}^{2 n}\left[W_{0 n}-w_{0 n}+(n-2) r_{0}^{2-2 n} \bar{x}_{0, n-2}\right], \\
& \bar{z}_{0 n}=r_{1}^{2 n}\left[Y_{1 n}-M_{1} w_{1 n}-\left(1+N_{1}\right) w_{0 n}+(n-2) r_{1}^{2-2 n} \bar{x}_{0, n-2}\right], \\
& \bar{z}_{i n}=r_{i}^{2 n}\left[W_{i n}-\left(1+M_{i}\right) w_{i n}-N_{i} w_{i-1, n}+(n-2) r_{i}^{2-2 n} \bar{x}_{i, n-2}\right], \\
& y_{0, n-2}=r_{0}^{4-2 n}\left[\bar{X}_{0, n-2}-n r_{0}^{2 n-2} w_{0 n}-\bar{x}_{0, n-2}\right], \\
& y_{0, n-2}=r_{1}^{4-2 n}\left[\bar{Z}_{1, n-2}-M_{1} \bar{x}_{1, n-2}-\left(1+N_{1}\right) \bar{x}_{0, n-2}-n r_{1}^{2 n-2} w_{0 n}\right], \\
& y_{i, n-2}=r_{i}^{4-2 n}\left[\bar{X}_{i, n-2}-\left(1+M_{i}\right) \bar{x}_{i, n-2}-N_{j} \bar{x}_{i-1, n-2}-n r_{i}^{2 n-2} w_{i n}\right] .
\end{aligned}
$$

For the case of the temperature a function of the radius the functions become very simple with the series in Eqs. (8) and (9) reducing to one term. With the temperature as $T_{i}(r)$ in $S_{i}$, the particular integral of $\nabla^{2} V=k T$ yields

$$
\begin{aligned}
& z \bar{Y}_{m}=k_{m} \int_{0}^{r} r T_{m}(r) d r, \\
& z \bar{Y}_{i}=k_{m} \int_{0}^{r_{m}} r T_{m}(r) d r+k_{m-1} \int_{r_{m}}^{r_{m-1}} r T_{m-1}(r) d r+\cdots+k_{i} \int_{r_{j+1}}^{r} r T_{i}(r) d r,
\end{aligned}
$$

whence, using Eq. (9), $[Y]_{i}=L_{i} t=A_{i 1} t=[Y]_{i-1}=E_{i 1} t$ with $r_{j}^{2} L_{i}=\left[z \bar{Y}_{i}\right]_{r=r_{j}}$. Further, $\phi_{j}(z)=a_{j 1} z$ and $\psi_{i}(z)=g_{i 1} / z$, where $a_{i 1}$ and $g_{i 1}$ are given by Eq. (10). The function $U$ is $U_{i}=a_{i 1} r^{2}+g_{i 1} \log r$ and the radial and tangential stresses in polar coordinates are

$$
\begin{aligned}
& \sigma_{r i}=2 a_{i 1}+\left(g_{i 1}-z \bar{Y}_{i}\right) / r^{2}, \quad \tau_{r \theta j}=0, \\
& \sigma_{\theta j}=2 a_{i 1}-\left(g_{i 1}-z \bar{Y}_{i}\right) / r^{2}-k_{i} T_{j} \\
& \sigma_{z i}=E_{j} e_{z}-k_{i} T_{j}+4 a_{i 1} / q_{j} .
\end{aligned}
$$

\section{ON THIRD-ORDER CORRELATION AND VORTICITY IN ISOTROPIC TURBULENCE*}

BY F. N. FRENKIEL (Cornell University)

The fundamental equation of the propagation of the correlation function obtained by Th. von Kármán and L. Howarth ${ }^{1}$ for isotropic turbulence in an incompressible viscous fluid, is written

$$
\frac{\partial\left(\overline{u^{\prime 2}} f\right)}{\partial t}+2\left(\overline{u^{\prime 2}}\right)^{3 / 2}\left(\frac{\partial h}{\partial r}+\frac{4}{r} h\right)=2 \overline{v u^{\prime 2}}\left(\frac{\partial^{2} f}{\partial r^{2}}+\frac{4}{r} \frac{\partial f}{\partial r}\right) .
$$

*Received Nov. 19, 1947.

'Th. von Kármán and L. Howarth. On the statistical theory of isotropic turbulence, Proc. Roy. Soc. London (A) 164, 192 (1938). 